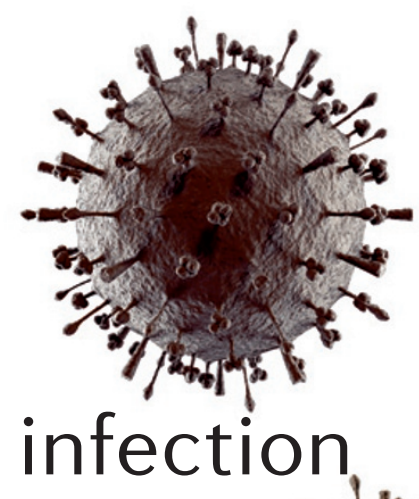

\title{
Novel agents fight flu infection
}

inhibitory concentration) values comparable to those of zanamivir and oseltamivir for the parent virus strains and considerably superior inhibition of resistant strains.

In cell culture, all DFSAs inhibited virus replication without causing cytotoxicity, performing comparably against influenza A strains, whereas FeqGuDFSA exhibited the highest potency of all inhibitors, including zanamivir, in cells infected with an influenza B strain. Importantly, the DFSAs were effective in vivo: all mice were protected from death following twice-daily intranasal treatment with FaxGuDFSA (a guanidine derivative containing an axial fluorine) or FeqGuDFSA beginning 2 hours prior to infection with a lethal influenza A strain.

Meanwhile, Morita and colleagues set out to investigate whether bioactive lipid mediators within the host, which are known to have antiinflammatory properties, might be involved in influenza virus infections.

First, they screened omega-3 polyunsaturated fatty acid-derived lipids in human lung epithelial cells that had been infected with an influenza A H1N1 virus strain, PR8, and found the docosahexaenoic acidderived protectin D1 isomer (PD1), 17S-dihydroxydocosahexanoic acid, to potently inhibit virus replication. This effect was confirmed in cells infected with the highly pathogenic $\mathrm{H} 5 \mathrm{~N} 1$ virus. Interestingly, analysis of the metabolic profiles of mice suffering from a severe PR8 or avian H5N1 infection revealed that endogenous production of several lipid mediators, particularly PD1, was reduced in the lungs, with levels of PD1 inversely correlating with the pathogenicity of different $\mathrm{H} 5 \mathrm{~N} 1$ virus isolates.
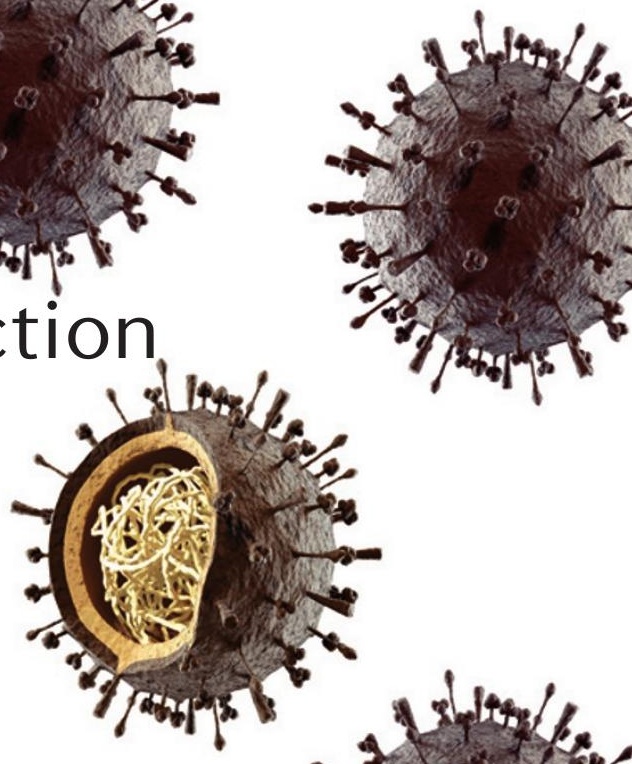

Therapeutically, intravenous administration of PD1

-12 hours before and immediately after PR8 or 2009 H1N1 virus infection - significantly improved the survival of mice. Notably, PD1 also demonstrated the potential to extend the therapeutic window of existing antiviral agents; although PD1 and the NA inhibitor peramivir had little effect on survival if given 2 days after infection with PR8, the combination of the two agents rescued all mice from death.

The beneficial effects of PD1 on survival were not due to antiinflammatory actions; PD1 was found to act by inhibiting the recruitment of viral RNAs to the mRNA transporter NXF1 (nuclear RNA export factor 1) to attenuate nuclear export and replication of viral RNA.

In summary, these studies identify novel potential agents and therapeutic strategies for the treatment of a broad spectrum of influenza strains, with the potential to address some of the limitations of existing agents.

Sarah Crunkhorn

ORIGINAL RESEARCH PAPERS Kim, J.-H. et al.

Mechanism-based covalent neuraminidase inhibitors with broad-spectrum influenza antiviral activity. Science 340, 71-75 (2013) | Morita, M. et al. The lipid mediator protectin D1 inhibits influenza virus replication and improves severe influenza. Cell 153, 112-125 (2013) 